\title{
A FOUR-VERTICES THEOREM FOR RULED SURFACES
}

\author{
P. JHA
}

ABSTRACT. The skewness of distribution of an orientable closed ruled surface satisfies a four-vertices theorem.

The concept of the skewness of distribution $\mu$ of a generator of a ruled surface was introduced by V. Rangachariar [1]. It is well known that $\mu$ is an invariant in the Euclidean geometry of ruled surfaces. In this paper, it is shown that $\mu$ has at least four extrema when it is associated to a certain curve on a unit sphere.

An oriented straight line in $E^{3}$ can be represented by a dual vector

$$
\mathbf{A}=\mathbf{a}+\tau \tilde{\mathbf{a}}
$$

where $\mathbf{a}$ is a unit vector along the straight line, $\widetilde{a}$ is the moment of a about the origin of coordinates $O$ and $\tau$ is an indeterminate subject to the relation $\tau^{2}=0$. By the definitions, $\mathbf{a}^{2}=1, \mathbf{a} \cdot \tilde{\mathbf{a}}=0$.

A ruled surface is represented by a curve on the dual unit sphere $[2, \S 8-2]$

$$
\mathbf{A}_{1}=\mathbf{A}_{1}(u)=\mathbf{a}_{1}(u)+\tau \tilde{\mathbf{a}}_{1}(u), \quad u_{0} \leq u \leq u_{1},
$$

where $u$ is a real parameter. The $\mathbf{A}(u)$ are identified with the generators of the surface. A closed ruled surface is represented on the dual unit sphere either by a closed curve or by a curve for which $\mathbf{A}\left(u_{1}\right)=-\mathbf{A}\left(u_{0}\right)$ since $\mathbf{A}$ and - A represent the same line. In the first case, we say that the ruled surface is orientable, in the second case nonorientable.

Let $S$ be a smooth, ruled surface generated by $A_{1}$. With each generator $A_{1}$ we associate an orthonormal trihedron determined by the generator, the normal to the ruled surface and the tangent to the ruled surface at the central point of the generator. Let $A_{1}, A_{2}, A_{3}$ be the dual vectors of the three edges of this trihedron. Then [2, p. 166]

$$
\mathbf{A}_{1}^{\prime}=K \mathbf{A}_{2}, \quad \mathbf{A}_{2}^{\prime}=-K \mathbf{A}_{1}+T \mathbf{A}_{3}, \quad \mathbf{A}_{3}^{\prime}=-T \mathbf{A}_{2},
$$

where primes denote differentiation with respect to $u$. We follow the notation of [2] and put

then

$$
K=k_{1}+\tau k_{2}, \quad T=t_{1}+\pi t_{2}
$$

$$
\mathbf{a}_{1}^{\prime}=k_{1} \mathbf{a}_{2}, \quad \mathbf{a}_{2}^{\prime}=-k_{1} \mathbf{a}_{1}+t_{1} \mathbf{a}_{3}, \quad \mathbf{a}_{3}^{\prime}=-t_{1} \mathbf{a}_{2}
$$

Received by the editors May 24, 1974 and, in revised form, August 1, 1974. AMS (MOS) subject classifications (1970). Primary 53A25. theorem.

Key words and phrases. Ruled surface, skewness of distribution, four-vertices 
Also,

$$
\mu=\operatorname{det}\left(\mathbf{a}_{1}, \mathbf{a}_{1}^{\prime}, \mathbf{a}_{1}^{\prime \prime}\right) \cdot\left|\mathbf{a}_{1}^{\prime}\right|^{-3}=t_{1} / k_{1} .
$$

Let $S$ be an orientable, closed, ruled surface.

Then $\mathbf{A}_{i}\left(u_{1}\right)=\mathbf{A}_{i}\left(u_{0}\right), i=1,2,3$, and

$\boldsymbol{\varphi}_{S} \mathbf{a}_{1} d \mu=\int_{u_{0}}^{u_{1}} \mathbf{a}_{1} d\left(t_{1} / k_{1}\right)=-\int_{u_{0}}^{u}\left(t_{1} / k_{1}\right) d \mathbf{a}_{1}=-\int_{u_{0}}^{u_{1}} t_{1} \mathbf{a}_{2} d u=\oint_{S} d \mathbf{a}_{3}=0$.

A curve on the surface of a sphere is called spherically convex if (a) it does not contain a pair of antipodal points and (b) it is the intersection of the sphere and a convex cone whose apex is the centre $O$ of the sphere.

Let the vector $a_{1}(u)$ generate a spherically convex closed curve $C$ on the unit sphere with centre $O$. We will show now that the function $\mu$ has at least four extrema on $C$. As it is continuous it has extreme values. We will show following [3] that it cannot have only two extrema.

Let $m$ and $M$ be the points on $C$ where this function has minimum and maximum values respectively. Let the function be increasing along one arc and decreasing on the other arc joining $m$ and $M$. Therefore, along one arc, $d \mu>0$ and along the other, $d \mu<0$. The equation of the plane $O m M$ is $\mathbf{a}_{1} \cdot \mathbf{n}=0$ where $\mathbf{n}$ is a constant vector. Hence $\mathbf{a}_{1} \cdot \mathbf{n}>0$ on one side of the plane and $\mathbf{a}_{1} \cdot \mathbf{n}<0$ on its other side. Therefore, let us say that $\mathbf{a}_{1} \cdot \mathbf{n}>0$ at points on the open $\operatorname{arc} d \mu>0$ and $\mathbf{a}_{1} \cdot \mathbf{n}<0$ at points on the open arc $d \mu<0$.

Hence we find that $\left(\mathbf{a}_{1} \cdot \mathbf{n}\right) d \mu>0$ for points on $C$ on both sides of the plane $O m M$ and consequently $\oint_{S}\left(\mathbf{a}_{1} \cdot \mathbf{n}\right) d \mu>0$. But

$$
\int_{S}\left(\mathbf{a}_{1} \cdot \mathbf{n}\right) d \mu=\left[\boldsymbol{\oint}_{S} \mathbf{a}_{1} d \mu\right] \cdot \mathbf{n}=0
$$

as $\mathbf{n}$ is a constant vector.

Thus we have a contradiction. Hence the function $\mu$ has more than two extrema. We have proved the following theorem:

If the unit vectors along the generators of a $C^{2}$, orientable ruled surface induce a spherically convex closed curve $C$ on the unit sphere, then the skewness of distribution has at least four extrema on $C$.

The author is grateful to Professor H. W. Guggenheimer for his kind guidance.

\section{REFERENCES}

1. V. Rangachariar (Chariar), Note on transversals which meet consecutive generators of a ruled surface at a constant angle, Bull. Calcutta Math. Soc. 37 (1945), 133-136. MR 7, 392.

2. H. W. Guggenheimer, Differential geometry, McGraw-Hill, New York, 1963. MR 27 \#6194.

3. - Geometrical applications of integral calculus, Lectures on Calculus, edited by K. O. May, Holden-Day, San Francisco, Calif., 1967. 